\title{
New Admission Control Policy for a Flexible VoLTE Service
}

\author{
Younes Labyad ${ }^{1,3}$, Mohamed Moughit ${ }^{1,2}$, Abderrahim Marzouk ${ }^{1}$, Noureddine Aboutabit ${ }^{1,2}$, \\ Abdelkrim Haqiq ${ }^{1,3}$ \\ Computer, Networks, Mobility and Modelling laboratory \\ Hassan 1st University \\ ${ }^{1}$ Faculty of Sciences and Techniques \\ Casablanca Street, Box 577, Settat, Morocco \\ ${ }^{2}$ National School of Applied Sciences \\ Béni Amir Street, Box 77, Khouribga, Morocco \\ ${ }^{3}$ e-NGN research group, Africa and Middle East
}

\begin{abstract}
LTE is the most recent mobile technology deployed everywhere in the whole wide world. It provides high Downlink and Uplink throughput for all service classes especially for voice class. All the services offered by LTE are based on IMS architecture. The goal of this paper is to present the developed admission control algorithm for VoLTE based on the Load control information and the UE capability in term of the supported voice codec. This developed algorithm has high performance results compared to the existing one that uses only G.711.
\end{abstract}

\section{General Terms}

Performing VoLTE quality of services is the goal of our paper.

\section{Keywords}

VoLTE, UE, AC, IMS.

\section{INTRODUCTION}

Even the LTE deployment is increasing faster in the world, it is still in need of development because of the usage of a full IP architecture for all classes of services especially for the voice service. This requires high quality service, because the QoS is directly perceived by the end user.

In this paper, we will try to understand the AC functionality in the E-NodeB and the relation with RRM entity especially with LC.All AC decision is based on LC.

In LTE and all previous technology the most important service that requires higher priority is the voice call. The AC rejection impacts directly other services such as streaming and FTP with dropping packets, rejecting new users and eventually downgrading the service.

Our idea in this paper is to involve the voice service in AC decision without decreasing the required quality of service based on used codec.
This paper is organized as follows. Next section describes the VoLTE architecture. The second one explains the admission control functionality. The fourth section presents the proposed admission control algorithm. Finally our algorithm results are presented and compared to the existing $\mathrm{AC}$ algorithm.

\section{Voice over Long term evolution}

\subsection{Overview}

Voice over LTE or VoLTE is a GSMA profile of the standard definition of the delivery of services currently provided via Circuit Switch networks - mainly voice and SMS - over the Packet Switched only network of LTE, leveraging the core network IP Multimedia Sub-System (IMS). When mobile networks deploy LTE radio access technology, conforming to the VoLTE profile provides operators with the assurance of interworking between their LTE network and the devices that connect to it, as well as providing the expected user the experience of voice Multi-Media Telephony service and SMS. In combination with Policy Control, IMS provides the required QoS appropriate for voice service using LTE radio access technology, thereby providing the user experience of voice calls that the subscribers expect. Moreover, VoLTE is designed to fully integrate with the existing user experience that is currently implemented in circuit switched voice devices. Therefore, even the call is a circuit switched call or a VoLTE call, it is transparent for the end user [1] [4].

\subsection{General architecture}

In the VoLTE the most important part is the VoLTE UE, LTE component including E-UTRAN and EPC, the IMS part including CSCF: Call Session Control Function that have three entity P-CSCF ( P for Proxy), S-CSCF ( S for Servin) and the application server that include Telephony and SMS application server. The figure. 1 show the VoLTE architecture [2] [4]. 


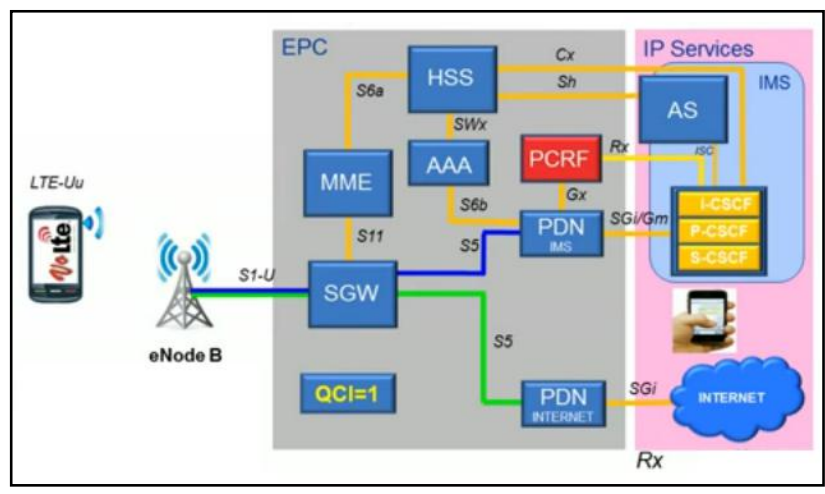

Fig.1: VoLTE Architecture

\subsection{VoLTE principal Nodes Description}

The functional nodes of the VoLTE architecture are defined by 3GPP and are described below. Further information can viewed in 3GPP TS 23.002 [1].

\subsubsection{VoLTE UE (User Equipment)}

The User Equipment that is used to connect to the EPC, in the figure 1bove this is an LTE capable UE accessing EPC via the LTE-Uu radio interface.

\subsubsection{Evolved Universal Terrestrial Access Network (E-UTRAN)}

The EUTRAN consists of a single node, the eNodeB that interfaces with the UE.

\subsubsection{Evolved Packet Core}

\subsubsection{MME (Mobility Management Entity)}

The Mobility Management Entity (MME) is the key controlnode for the LTE access network.. The MME provides the control plane function for mobility between LTE and 2G/3G access networks and interfaces with the home HSS for roaming UEs.

\subsubsection{SGW (Serving Gateway)}

The SGW routes and forwards user data packets, while also acting as the mobility anchor for the user plane during intereNodeB handovers and as the anchor for mobility between LTE and other 3GPP technologies (terminating the S4 interface and relaying the traffic between $2 \mathrm{G} / 3 \mathrm{G}$ systems and PGW).

\subsubsection{IMS}

IMS is the control infrastructure for supporting next generation IP Multimedia Services and consists of many separate elements which are listed below.

\subsubsection{P-CSCF (Proxy Call Session Control Function)}

The P-CSCF is the initial point of contact for session signalling for the IMS-enabled VoLTE UE. The P-CSCF behaves as a SIP proxy by forwarding SIP messages between the UE and the IMS Core Network, maintains the security associations between itself and the VoLTE UE, and incorporates the Application Function aspect of PCC to enable binding of the IMS session with the bearer for applying dynamic policy and receiving notifications of bearer level events. The P-CSCF may be implemented in an Access Session Border Controller which may also incorporate the IMS-ALG/IMS-AGW [2].

\subsubsection{I-CSCF (Interrogating Call Session Control Function)}

The I-CSCF is the contact point within an operator's network for all connections destined to a user of that network. On IMS registration, it interrogates the HSS to determine which suitable S-CSCF to route the request for registration. For mobile terminating calls, it interrogates the HSS to determine which S-CSCF the user is registered on [2] [4].

\subsubsection{S-CSCF (Serving Call Session Control Function)}

The S-CSCF provides session set-up, session tear-down, session control and routing functions. It generates records for billing purposes for all sessions under its control, and invokes Application Servers based on IFCs received from the HSS. The S-CSCF acts as SIP registrar for VoLTE UEs that the HSS and I-CSCF assign to it. It queries the HSS for the applicable subscriber profiles and handles calls involving these end points once they have been registered [2]

\subsubsection{Telephony Application Server (TAS)}

The TAS is an IMS Application Server providing support for a minimum set of mandatory MultiMedia Telephony (MMTel) services as defined by $3 \mathrm{GPP}$

\section{Admission control in VoLTE}

A bearer is an IP packet flow with a defined Quality of Service (QoS). E-UTRAN Radio Access Bearer (E-RAB) refers to the concatenation of an $\mathrm{S} 1$ bearer and the corresponding radio bearer. LTE uses the concept of ERAB to route the packets of an Evolved Packet Core (EPS) bearer between a serving gateway (S-GW) and a user equipment (UE). The admission control tasks in LTE is to decide whether or not to establish new E-RAB. The figure 2 show the admission control process [3] [6]

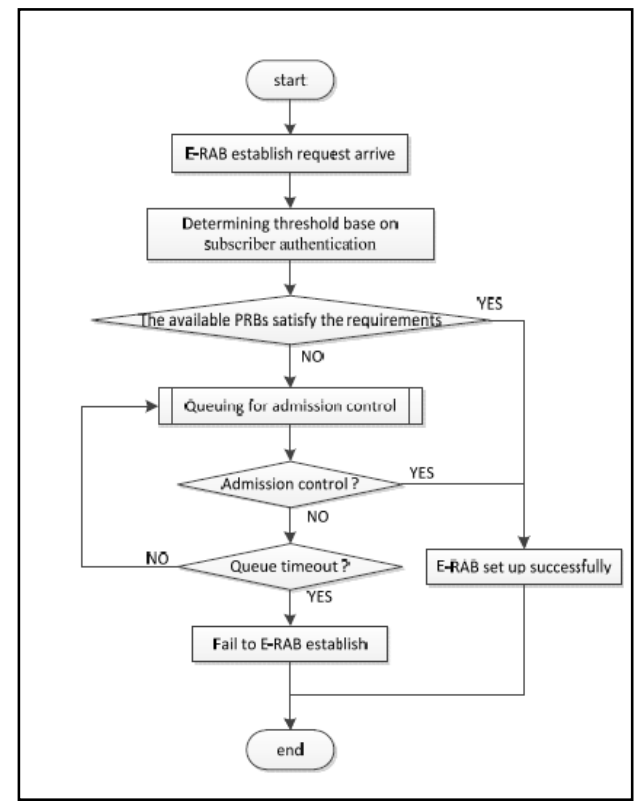

Fig.2: Admission control process

Firstly the admission control entity define the number of necessary PRB for requested service by the mobile in the time t. in the RRC phase we note this by $P_{R B}{ }_{\text {req }}^{t}$ after the estimation and based on Load control information the admission control algorithm compare the available PRB $\left(\mathrm{PRB}_{\text {Avail }}^{\mathrm{t}}\right)$ with $\mathrm{PRB}_{\text {req }}^{\mathrm{t}}$ after this comparing the AC take the decision to admit or reject the new RAB [3] [6]. 
The formula that define $\mathrm{PRB}_{\text {Avail }}^{\mathrm{t}}$ in the time $\mathrm{t}$ is preseted by Formula.1 :

$$
\text { PRB }^{\mathrm{t}}{ }_{\text {Avail }}=\mathrm{PRB}_{\text {total }}-\mathrm{PRB}^{\mathrm{t}} \text { Used }
$$

The PRB ${ }_{\text {total }}^{t}$ is the PRB that the E-NodeB can provide based on the total power, used frequency band and the number of carrier and $\mathrm{PRB}^{\mathrm{t}}$ Used is the used PRB in the time $t$ by all the service including Real time Services and No Real Time service and also signaling PRB. The used PRB presented in the Formula.2

$$
P R B_{\text {used }}^{t}=P R B^{t}{ }_{\text {Used-RT }}+P B^{t}{ }_{\text {Used-NRT }}+\text { PRB }_{\text {Used-Sig }}^{t}
$$

The admission control accept a RAB setup when $\mathrm{PRB}^{\mathrm{t}}{ }_{\text {Avail }}<$ $\mathrm{PRB}^{\mathrm{t}}$ req and reject the call in the other case.

\subsection{Proposed AC algorithm for voice call}

The existing admission control algorithm doesn't include RT service To decide the rejection or not of the new call.In fact, the real time services have the high priority, but as we know all the new smartphones can support other codec than G.711 as recommended by the IUT. So, thus our idea in the case of overload is to admit new RT user with other codec.

When we have voice call the recommended codec by the IUT is the G.711 due to its high quality of service. However, in other case when the load of the E-NodeB is high we can use G.729 or G.723 that require less number of PRB.

The system Load is defined by Formula. 3

$$
\text { SYS }_{\text {load }}^{\mathrm{t}}=\left(\mathbf{P R B}_{\text {Used }}^{\mathrm{t}}+\mathrm{PRB}_{\text {req }}^{\mathrm{t}}\right) / \mathrm{PRB}_{\text {total }}
$$

In our proposal we define 3 threshold before starting call rejection:

Feasible Load Area $\left(0 \%<\mathbf{S Y S}_{\text {load }}<=60 \%\right)$ :in this AREA we can use G.711 or the other codec but the priority is given to G.711

Preventive Load Area $\left(\mathbf{6 0} \%<\mathbf{S Y S}_{\text {load }}<\mathbf{= 7 5 \% )}\right.$ : in This area the system is not overloaded but we must use G.729 because contuning using G.711 it can overload the system early.

Overloaded Area $\left(\mathbf{7 5 \%}<\mathbf{S Y S}_{\text {load }}<\mathbf{= 9 5 \% )}\right.$ ): The prefered codec is G.723 because the system is overloaded and G.711 and G.729

Rejection Area $\left(\mathbf{9 5 \%}<\mathbf{S Y S}_{\text {load }}<=\mathbf{1 0 0 \%}\right)$ : In this aree AC start rejecting call without taking care of used codec.

After defining every AREA the AC process is summarized in Figure 3:

\subsection{Performance Evaluation}

The Admission control algorithm was performed with $\mathrm{C}++$ in order to evaluate the voice performance and the system load when all new calls use only one of the codec without taking care of the load status provided by Load control entity and with developed algorithm when we divise the EnodeB load into 4 area and the system start rejecting until reaching $95 \%$.

In the simulation We consider an LTE system with $5 \mathrm{MHz}$ of downlink channel bandwidth which is one of the channel bandwidths specified in LTE system that mean that we have 50 PRBs per $1 \mathrm{~ms}$ TTI and also we use 64QAM. The table 1 showed the detail of LTE system.

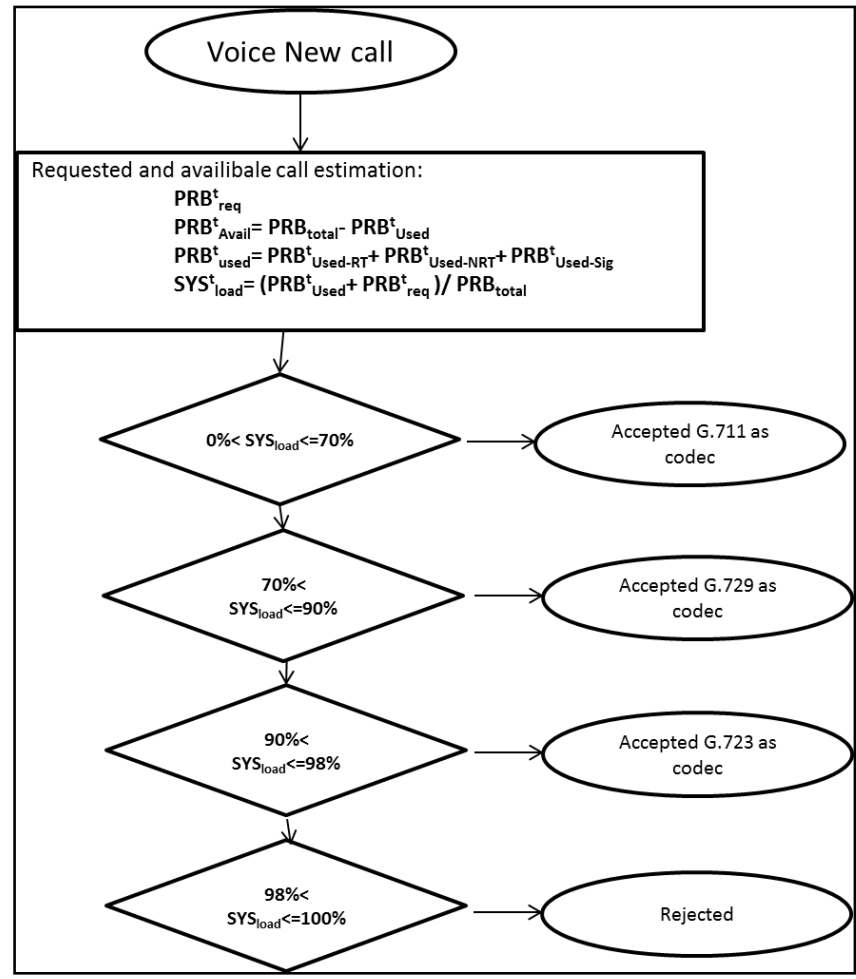

Fig.3: Proposed Admission Control Algorithm

The table 2 shows the initial simulation condition that we are using, 3 service are used : FTP with 5 connexion of $1 \mathrm{Mbps}$, Video with 5 connexion of $1 \mathrm{Mbps}$ each and $120 \mathrm{VoLTE}$ connxions.

\begin{tabular}{|l|l|}
\hline \multicolumn{1}{|c|}{ Parameter } & \multicolumn{1}{c|}{ Value } \\
\hline Downlink channel bandwidt & $5 \mathrm{MHz}$ \\
\hline PRB & $50 \mathrm{PRB}$ per TTI \\
\hline TTI & $1 \mathrm{~ms}$ \\
\hline Modulation & $64 \mathrm{QAM}$ \\
\hline CQI & 12 \\
\hline bits per RB & $562 \mathrm{bits}$ \\
\hline Throughput & $28.1 \mathrm{Mbps}$ \\
\hline
\end{tabular}

Table.1: Proposed Admission Control Algorithm

\begin{tabular}{|l|l|}
\hline \multicolumn{1}{|c|}{ Service } & \multicolumn{1}{c|}{ Number of connexions } \\
\hline FTP & $5 * 1 \mathrm{Mbps}$ \\
\hline Video & $5 * 1 \mathrm{Mbps}$ \\
\hline VoLTE & 120 connexions \\
\hline
\end{tabular}

Table.2: initial system status

The first step will analyze the result of the load with the normal LTE AC algorithm using G.711 and also using the new algorithm when we use the codecs G.711, G.729 and G.723 but every codec used after that the load reach a threshold. The finding result show that the necessary time for normal AC algorithm to reach $100 \%$ is 32 second but for the new algorithm it reach the over loaded area after about 227 second that mean in the busy hour with the new algorithm we can guaranty the system stability 7 time more than the normal codec. The figure 4 shows the comparing of ENB load. 


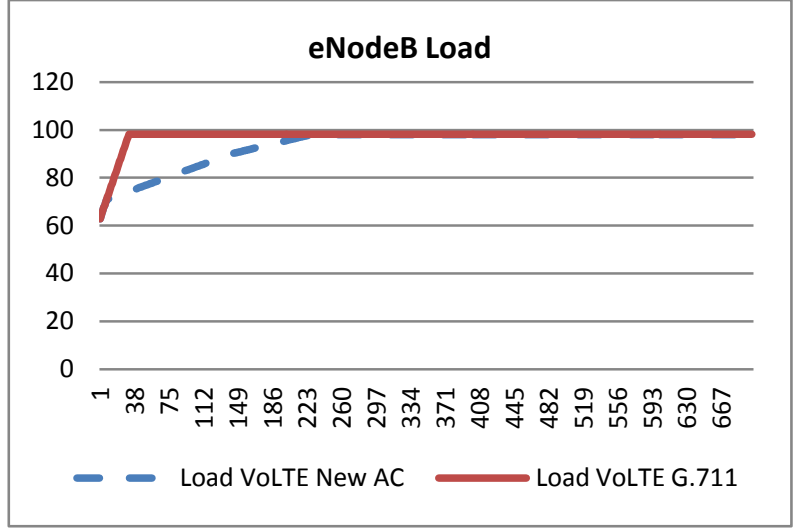

Fig.4: EnodeB Load comparing

The number of VoLTE users is increasing by using the new admission control algorithm because of Enode Load give more capacity to admit new voice users. The Figure 5 show the number of VoLTE by using the normal AC algorithm and the proposed one. We can observe that with the new algorithm give more number of user without impacting existing RT and NRT connexions.

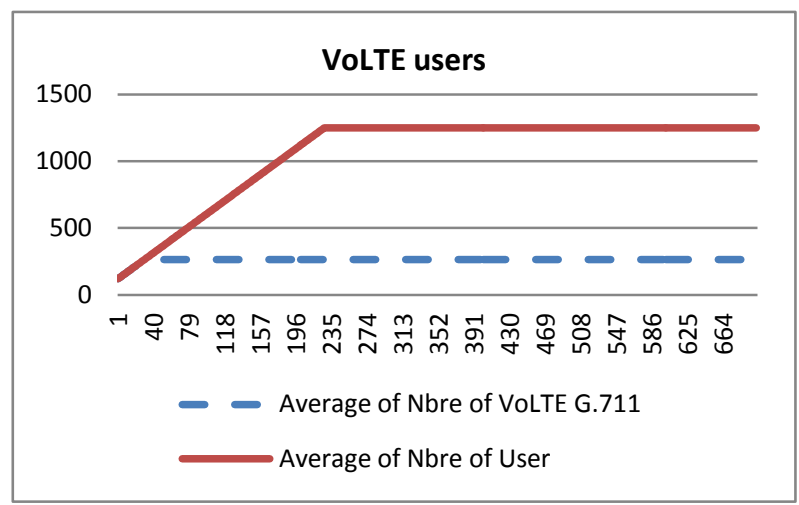

Fig.5: VoLTE users

The mean score opinion decrease when we use the new VoLTE AC In fact, the other codecs than G.711 doesn't have the same value as G.711. However, the obtained value can be considered as a good performance if we take into account that the new algorithm give 7 time high than the normal one.

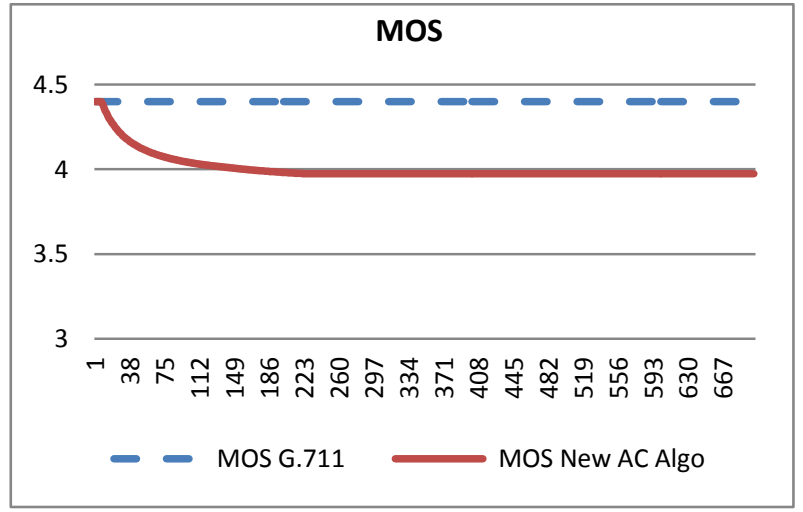

Fig.6: Mean score opinion

\section{CONCLUSION}

This paper present a new admission control algorithm for VoLTE user based on EnodeB load provided by load control entity. The result shows that the nevel algorithm is much better because it give more system capacity with good voice performance especially for Mean score opinion. Finally the research team aims to extend this work to provide a complete $\mathrm{AC}$ algorithm based on all kind of service.

\section{REFERENCES}

[1] M. R. Tabany and C. G. Guy, " Performance Analysis and Deployment of VoLTE Mechanisms over 3GPP LTE-based Networks," International Journal of Computer Science and Telecommunications, Vol. 4, Issue. 10, Oct. 2013, pp.1-8.

[2] 3GPP TS 23.002, Network Architecture, V10.2.0 (201103).

[3] A Resource-estimated Call Admission Control Algorithm in 3GPP LTE System, Sueng Jae Bae1, Jin Ju Lee, Bum-Gon Choi, Sungoh Kwon, and Min Young Chung

[4] Younes Labyad, Mohammed MOUGHIT and Abdelkrim HAQIQ, "Impact of using G.729 on the Voice over LTE performance", International Journal of Innovative Research in Computer and Communication Engineering,Vol. 2, Issue 10, October 2014

[5] Seung Que LEE, RYU Bying Han and Nam-Hoon PARK, "Call admission control for hybrid access mode femtocell system,”WiMob 2011, Oct. 2011, pp. 512-516.

[6] M. E. R. Khan, "Fair admission control policies for femtocell operation," ICUFN 2012, July 2012, pp. 175179.

[7] Siomina, I.; Wanstedt, S.; , "The impact of QoS support on the end user satisfaction in LTE networks with mixed traffic," IEEE 19th International Symposium on Personal, Indoor and Mobile Radio Communications, pp.1-5, 15-18 Sept. 2008.

[8] Zaki, Y.; Weerawardane, T.; Gorg, C.; Timm-Giel, A., "Multi-QoS-Aware Fair Scheduling for LTE," IEEE 73rd Vehicular Technology Conference (VTC Spring) vol., no., pp.1-5, 15-18 May 2011.

[9] 3GPP Technical Specification 23.203, "Policy and charging control architecture (Release11)", www.3gpp.org, 2012. 